\title{
Comparison of Energy Detection Methods in Cognitive Radio Networks
}

\author{
H. Srikanth Kamath \\ Dep. of Electronics and Communication Engg. \\ Manipal Institute of Technology \\ Manipal, India
}

\author{
Simon Larsson \\ Dep. of Electronics and Communication Engg. \\ Manipal Institute of Technology \\ Manipal, India
}

\begin{abstract}
Cognitive Radio is an emerging new paradigm in wireless communications. Its goal is to make frequency use more efficient by using temporarily unoccupied frequency bands. Therefore frequency bands have to be measured in order to decide about the occupation of the band. One of the used techniques is energy detection. In this paper different energy detection models were compared. The evaluation was performed using a simulator for an OFDM-modulation based wireless communication network.
\end{abstract}

\section{General Terms}

Cognitive Radio, Energy Detection Models, OFDM(A), Wireless Mobile Communication Network Simulator

\section{Keywords}

cognitive-radio; energy-detection; chi-squared; OFDM(A); cyclic-prefix; simulator

\section{INTRODUCTION}

Cognitive Radio (CR) is used in mobile communication to make devices and networks better adaptable to changes in environmental parameters such as frequency allocation and regulation. Therefore a CR device has to sense its surroundings in different ways. According to the obtained input parameters the output parameters of the mobile device are adapted. In the case studied here, the output parameter that is changed is the used frequency band. A general introduction into CR and related topics is given in [1]. A general overview is given in survey papers such as [2][3].

The available information from the sensors is combined in a module called spectrum manager (SM). The SM also decides about the output parameters. The SM module and some of its input parameters are shown in Figure 1. The input parameter considered in this paper is the spectrum sensing module that searches unoccupied frequency bands available for transmission. Additional information is obtained by Geolocation and database access in order to take into account the regularization of frequency allocation in certain areas. This insures that the mobile device only uses frequencies which it is allowed to use.

The output parameter, the frequency band to be used, is selected according to these input parameters. The dynamic spectrum access is obtained by adopting software defined radio techniques which allow a fast change of working frequency during operation.

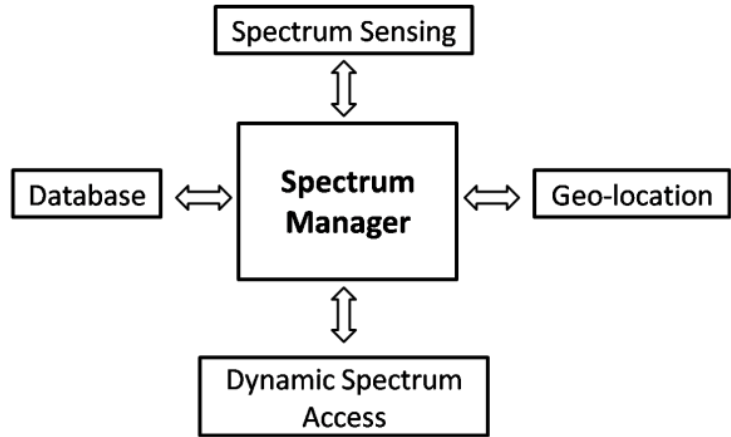

Fig 1: Schematic view of the spectrum manager

In the domain of spectrum sensing, several different techniques have been introduced in the literature. An overview is given in the book [1] and in [4][5][6][7].

In an interference temperature model the power available at the receiver is measured. The allowed interference temperature is limited by a certain interference temperature limit that is not to be exceeded. This approach was followed in numerous papers but was not considered for further investigation due to the fact that it causes more interference to the PU and the improvements to bandwidth utilization is low [1].

The spectrum sensing technique under consideration in this paper is energy detection. The basic concept of energy detection is introduced in the next section. After that the different models investigated and the simulator used in this paper are introduced. In the fifth section the results are given followed by conclusions and future research topics.

\section{ENERGY DETECTION}

For an energy detector based approach two receiver states are distinguished. In the first one, no primary user (PU) and thus only the additive white Gaussian noise (AWGN) $w(n)$ of the channel is present:

$$
\mathcal{H}_{0}: y(n)=w(n) .
$$

In the other case an additional PU is present. In this case $\mathrm{x}(\mathrm{n})$ is the signal of the PU:

$$
\mathcal{H}_{1}: y(n)=x(n)+w(n) .
$$

To observe the current state, the energy of the signal is evaluated using a function depending on the received signal $y(n)$ :

$$
T=f(y(n)) .
$$


If the value $T$ surpasses a certain threshold $\lambda$ the $P U$ is assumed as present. Else the SU is allowed to use the frequency band.

In order to evaluate the efficiency of the spectrum sensing, two probabilities are computed. The first one is the false alarm (FA) probability. It shows the probability that a PU is detected if there is actually none:

$$
P_{F A}=\operatorname{Pr}\left(T>\lambda \mid \mathcal{H}_{0}\right) .
$$

On the other hand the probability of missed detection (MD) is given as the times when there is a PU present but none is detected by energy detection:

$$
P_{M D}=\operatorname{Pr}\left(T<\lambda \mid \mathcal{H}_{1}\right)
$$

In the case of a false alarm a part of the spectrum remains unused. In the case of missed detection the transmission of the secondary user (SU) will cause interference to the PU. The goal is to define a threshold $\lambda$ that minimizes one of the probabilities $P_{F A}$ or $P_{M D}$.

\section{MODELS}

The resulting distribution of $\mathrm{T}$ can be modeled differently depending on the function $f(y(n))$ used in Eq. (3). The two different models implemented and evaluated in this paper are introduced in the following subsections.

\subsection{Gaussian Model}

One possibility of calculating $T$ is to compute the average energy over a certain observation interval $N[8]$ :

$$
T=\frac{1}{N} \sum_{n=1}^{N}|y(n)|^{2} .
$$

Considering this case the resulting distributions for the cases $\mathcal{H}_{0}$ and $\mathcal{H}_{1}$ respectively are shown in Figure 2 . In this case an appropriate model is given with two Gaussian distributions. One is centered at the noise level $\sigma^{2}$ whereas the other one is shifted by the energy of the data signal Es. Summarized the model is given as

$$
T \sim \begin{cases}\mathcal{N}\left(\sigma^{2}, \frac{1}{N} \sigma^{4}\right) & \mathcal{H}_{0}, \\ \mathcal{N}\left(E_{S}+\sigma^{2}, \frac{1}{N}\left(E_{s}+\sigma^{2}\right)^{2}\right) & \mathcal{H}_{1} .\end{cases}
$$

The resulting values for $\mathrm{T}$ can be accumulated in a histogram. This is shown for the Gaussian model in Figure 2. The graph of an absent PU is colored in green whereas the histogram is blue for the case where a PU is present. In red the curves according to the Gaussian model are plotted. The chosen threshold is shown as a vertical line.

\subsection{Chi-squared Model}

The second model is based on a modified formula for the calculation of $T$ where only the sum over the detected signal is computed [9]:

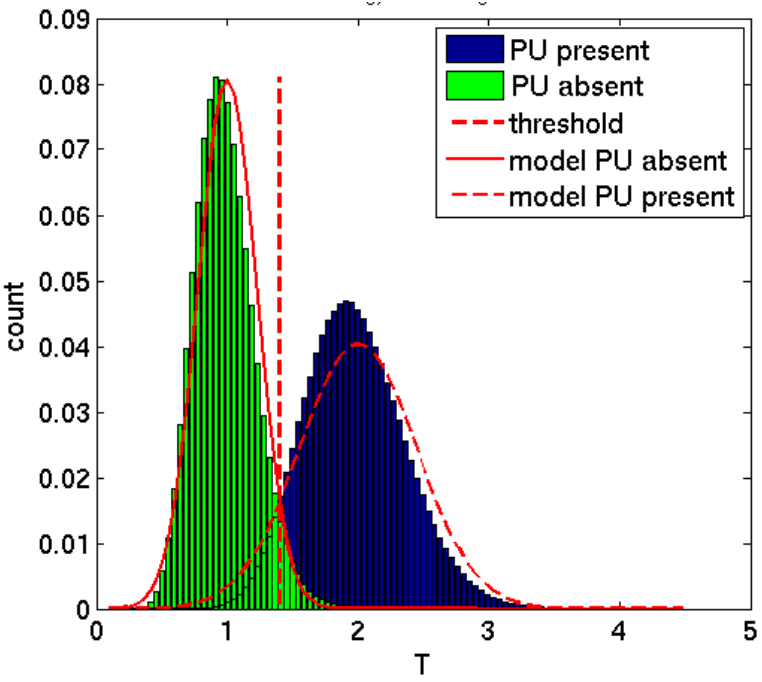

Fig 2: Energy level histogram for the Gaussian model

$$
T=\sum_{n=1}^{N}|y(n)|^{2} .
$$

In this case the resulting values for $T$ are Chi-squared distributed $\left(\chi_{k}^{2}\right)$. A Chi-squared distribution is described by one parameter $k$ usually referred to as degrees of freedom which is also the distribution's mean value. The parameter $k$ is given by the summation interval $N$.

In the case of $\mathcal{H}_{0}$, in which the PU is absent, the summation is acquired over the noise term $w(n)$ only. In this case the distribution of $\mathrm{T}$ can be modeled as $\chi_{N}^{2}$. In the case of a present PU the data signal term $w(n)$ is added to the signal $y(n)$, which leads to a rise in the detected energy by the signal-to-noise ratio (SNR) $E_{S} / N_{0}$. In this case the degrees of freedom and thus the mean value of the distribution modeling the $\mathcal{H}_{0}$ case is given with $k=\left(N_{0}+E_{s}\right) N$. The resulting distributions are summarized as follows:

$$
T \sim \begin{cases}\chi_{N}^{2} & \mathcal{H}_{0}, \\ \chi_{\left(N_{0}+E_{S}\right) N}^{2} & \mathcal{H}_{1} .\end{cases}
$$

The histogram with respect to the Chi-squared model is given in Figure 3. Like before the graph for absent and present PU are given in green and blue respectively. The computed curves according to this model are plotted in red. The threshold is marked as a vertical line.

\subsection{Threshold}

The threshold $\lambda$ on which the false alarm and missed detection probabilities are evaluated is computed based on these models. For each SNR, $\lambda$ is set to the intersection point of both theoretical curves. For both cases the equations are solved analytically and are implemented in the simulator. The simulator is described in detail in the following section.

\section{SIMULATOR}

In order to be able to compare the energy detection models a simulator for a wireless $\mathrm{CR}$ network was developed. The simulator is loosely based on the specification of the IEEE 802.22 network [10]. In this standard unoccupied frequency bands in the TV domain are used for a RAN wireless network. Based on this network occupied and unoccupied channels are sensed. 


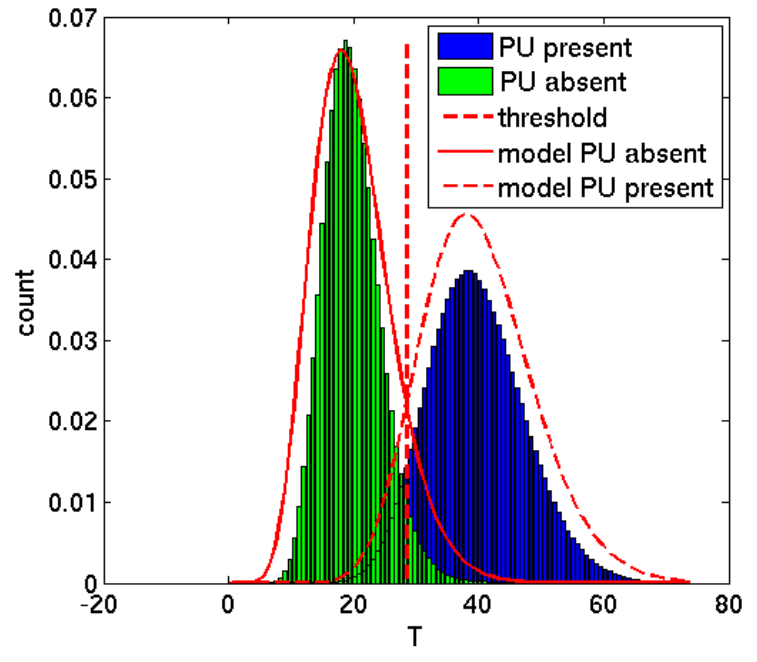

Fig 3: Energy level histogram for the Chi-squared model

The simulator uses a 2048 subcarrier OFDMA (Orthogonal Frequency-Division Multiple Access) modulation scheme. 1680 of the subcarriers are active. The remaining 368 subcarriers are used as left and right guard-bands. The blockdiagram of the simulator is given in Figure 8 .

Binary data are mapped to modulation symbols. The modulation schemes can be BPSK, QPSK or 16QAM. Consecutively the subcarriers are allocated. In the simulator the subcarriers are allocated after a given scheme such that there are occupied and unoccupied channels that can be measured. After that the OFDMA modulation is done by an inverse fast Fourier transformation (IFFT).

Before transmission a Cyclic Prefix (CP) is added to the data stream which is then given to the channel. The channel adds Gaussian noise to the signal (AWGN). In the receiver the CP is removed and the OFDMA modulation is reversed by a fast Fourier transformation (FFT). The subcarriers are deallocated and the symbols are demapped to reveal the original data.

The subcarriers are further processed in the spectrum sensing module. In this part of the simulator the energy detection is done. The results of the spectrum sensing are consecutively handed to the spectrum manager (SM). The SM uses the results of the spectrum sensing to decide whether a PU is present or not. Therefore it not only takes into account the sensing results but also other parameters. A simple model of the SM was shown in Figure 1.

The output of the SM is a hard decision of the occupancy of the OFDM subcarrier. From these results the histograms, false alarm and missed detection probabilities are computed. The results are discussed in the next section.

\section{RESULTS}

In this section the results of the simulations are shown. At first the operation of the receiver is considered. After that the performance of the spectrum manager is evaluated.

\subsection{BER evaluation}

In order to verify the simulator, the simulated BER values are compared to the theoretical curve in Figure 4. The results are plotted in a blue line whereas the theoretical values for a BPSK modulated OFDM system are given with red crosses. It can be seen that the simulator provides the BER results expected from an OFDM system.

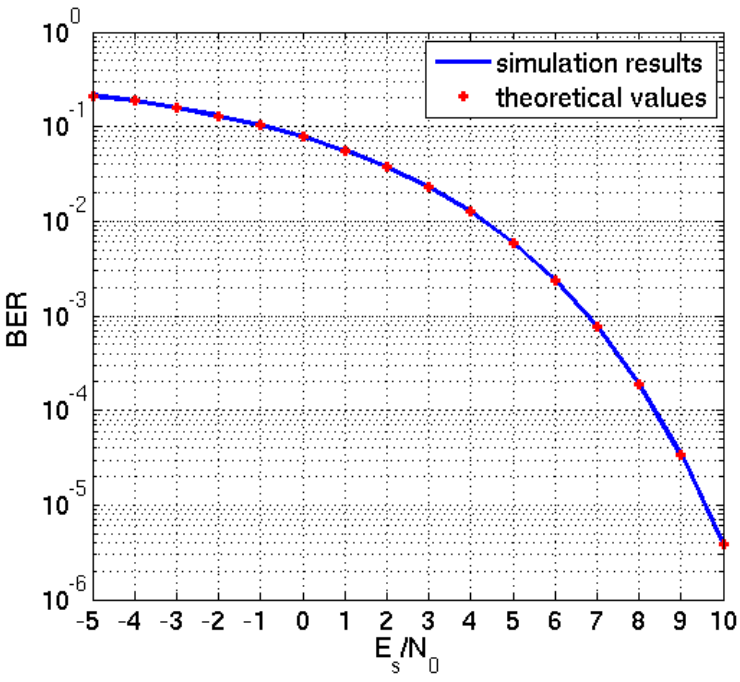

Fig 4: Bit error rate evaluation for BPSK modulation

\subsection{ROC evaluation}

The receiver operation characteristic (ROC) curves show the performance of a receiver. Therefore the probability of false alarm $P_{F A}$ is plotted over the probability of missed detection $P_{M D}$ acquired using different threshold values $\lambda$.

The ROC curves for both models are given in Figure 5. As one can see the receiver characteristic shows the desired low values for medium thresholds. The differences between the two models with respect to the ROC curves are very small.

\subsection{Missed detection evaluation}

In order to compare the two energy detection models the missed detection probability was evaluated for different SNR values. The results are given in Figure 6 where the graph for the Gaussian model is given as a dashed line whereas the Chisquared model is given as a solid graph.

At an SNR value below $-0.5 d B$ the Chi-squared model yields a lower probability of missed detection. Above this value the Gaussian model shows a lower value for missed detection. The missed detection probability thus decreases faster in the Gaussian model.

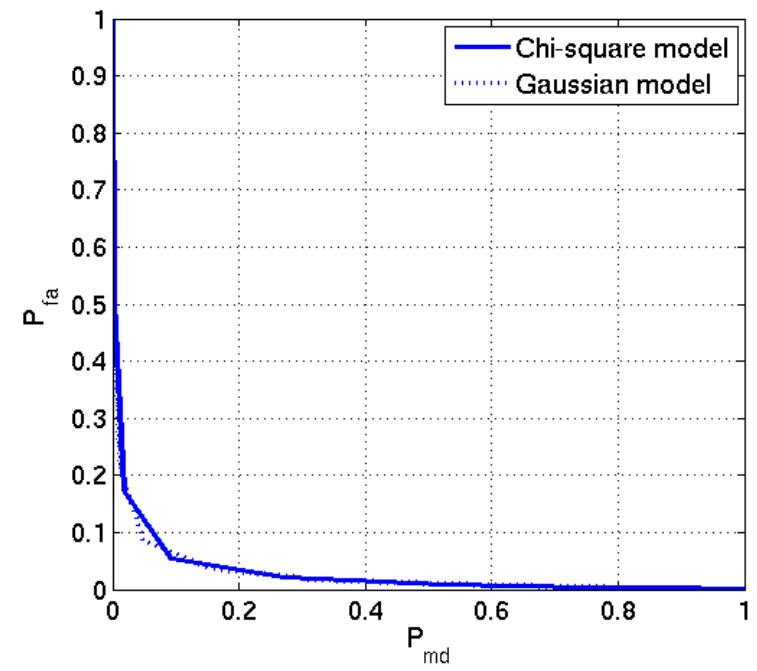

Fig 5: Receiver operation characteristics for both models 


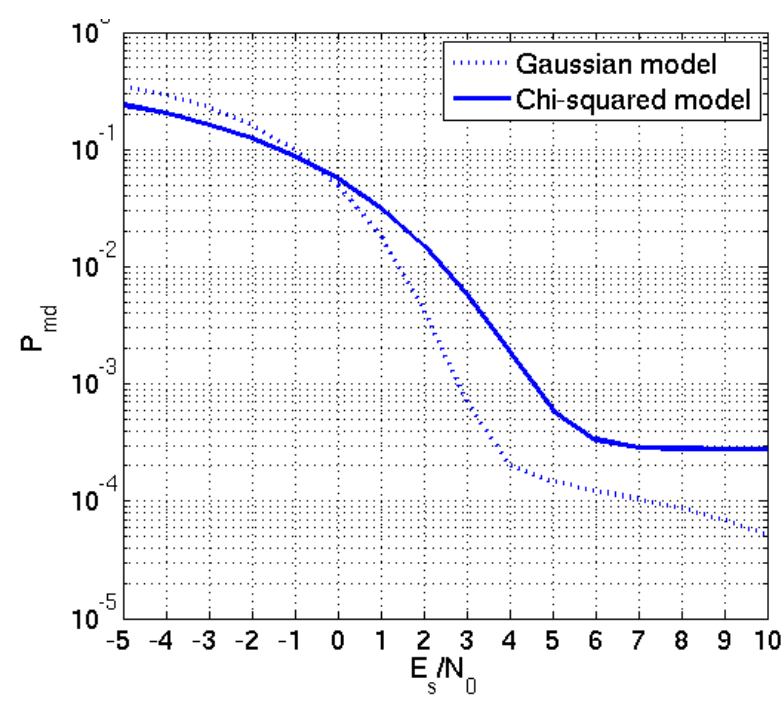

Fig 6: Comparison of missed detection probabilities between the two models

If the SNR surpasses a certain value the missed detection probability decreases slower. For the Gaussian model this value is at $4 d B$ whereas it is at $6 d B$ for the Chi-squared model.

In order to evaluate the saturation of the missed detection probability the simulation was repeated with a decreased simulation length. Whereas there were 12000 OFDM symbols simulated before now only 6000 symbols were simulated. The results are given in Figure 7 for the Gaussian model.

Since the total number of unoccupied channels is fixed, the number of missed detections rests at one level over a certain SNR value. As a consequence this saturation level drops if the number of OFDM symbols is raised. This can be seen in both models.

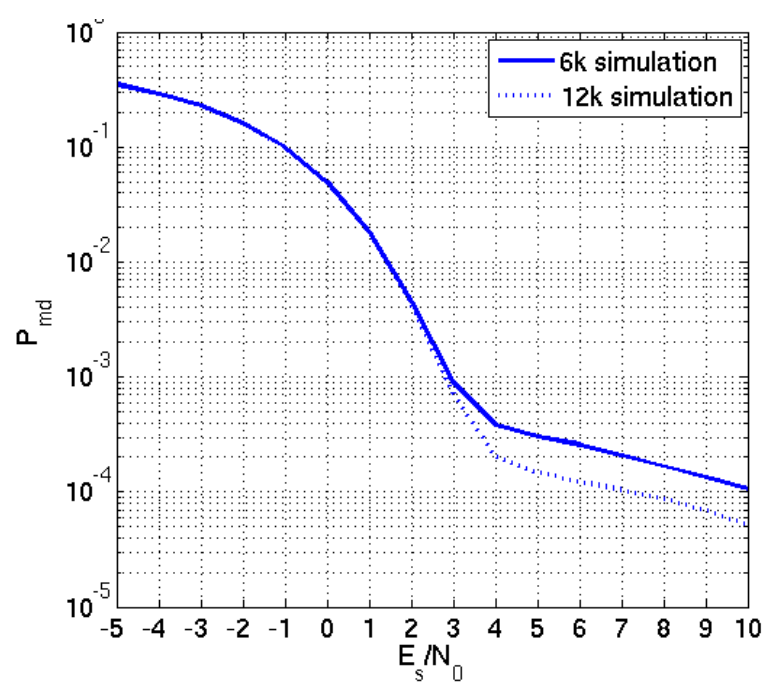

Fig 7: Simulation length comparison

\section{CONCLUSIONS}

Two cases for the modeling of energy detection in a cognitive radio network were compared. Therefore a simulator for an OFDM based network was implemented and evaluated by its BER values. The models compared were based on Gaussian distributions and Chi-squared distributions respectively. Based on these models a decision about the presence of a primary user was made. Therefore the decision threshold is set to the intersection point of the model functions. The results show that the Gaussian model is appropriate for high SNR values whereas the Chi-squared model yields a better performance at lower SNR levels. Both models showed similar ROC characteristics.

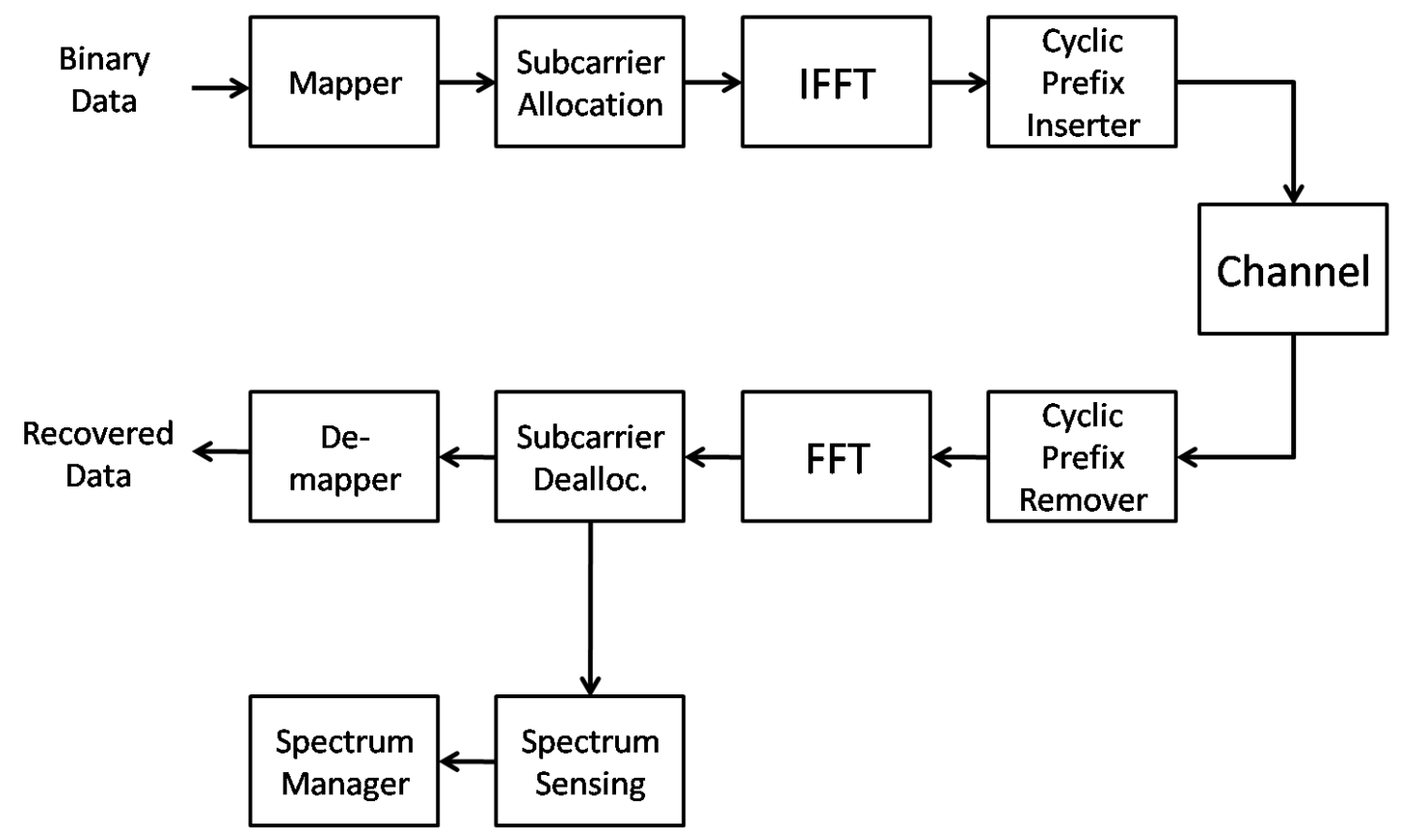

Fig 8: Block-diagram of the simulator 
For future investigations the simulator should be altered to random occupation patterns for the OFDM subcarrier allocation such that the saturation of the probability values is avoided.

Additionally there are several different models available in the literature which can be evaluated in future works. Interference temperature models can be implemented to evaluate the observation mentioned in the beginning. Different detection methods are given by feature based methods. In this case specific features of a PU's signal are used to decide whether a frequency band is occupied.

\section{REFERENCES}

[1] K. J. R. Liu and B. Wang. "Cognitive Radio Networking and Security". Cambridge : Cambridge University Press, 2011. 978-0-521-76231-1.

[2] L. Khalid and A. Anpalagan. "Emerging cognitive radio technology: Principles, challenges and opportunities". p. 358-366, Toronto : Computers and Electrical Engineering, 2010, Vol. 36.

[3] I.F. Akyildiz, W.-Y. Lee, M.C. Vuran and S. Mohanty. "NeXt gereration/dynamic spectrum access/cognitive radio wireless networks: A survey." 2006, Computer Networks 50, pp. 2127-2159.

[4] C. Raman, J. Kalyanam, I. Seskar and N. Mandayam. "Distributed Spatio-Temporal Spectrum Sensing: An
Experimental Study.” Pacific Grove, CA. IEEE, 2007. 978-1-4244-2110-7.

[5] F.F. Digham, M.-S. Alouini and M.K. Simon. "On the Energy Detection of Unknown Signals over Fading Channels." IEEE, 2003, Vols. Communications, 2003. ICC '03. IEEE International Conference on (Volume: 5). 0-7803-7802-4.

[6] H.-S. Chen, W. Gao and D.G. Daut. "Spectrum Sensing Using Cyclostationary Properties and Application to IEEE 802.22 WRAN." Washington, DC. IEEE, 2007. 978-1-4244-1043-9.

[7] M. P. Wylie-Green. "Dynamic Spectrum Sensing by Multiband OFDM Radio for Interference Mitigation". Baltimore : IEEE, 2005. 1-4244-0013-9.

[8] S. J. Shellhammer. "Spectrum sensing in IEEE 802.22". San Diego : Qualcomm Inc, 2008.

[9] T. Yücek and H. Arslan. "A Survey of Spectrum Sensing Algorithms for Cognitive Radio Applications". IEEE Communications Surveys \& Tutorials, 2009, Vol. 11.

[10] IEEE Computer Society. "Part 22: Cognitive Wireless RAN Medium Access Control (MAC) and Physical Layer (PHY) Specifications: Policies and Procedures for Operation in the TV Bands". IEEE Standard for Information Technology. New York : IEEE, 2011. 